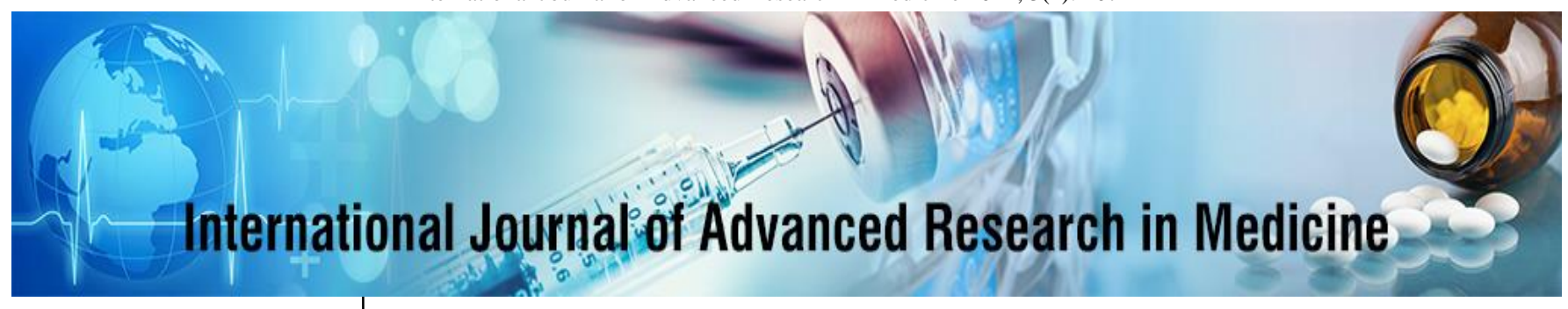

E-ISSN: 2706-9575

P-ISSN: 2706-9567

IJARM 2021; 3(2): 107-111

Received: 15-10-2021

Accepted: 10-11-2021

Dr. Spandana Vuyyuru Senior Resident, Department of Nephrology, Narayana Medical College, Nellore, Andhra Pradesh, India

Dr. K Vara Prasada Rao Associate Professor, Department of Nephrology, Narayana Medical College, Nellore, Andhra Pradesh, India

Dr. Moturu Venkata Viswanath Assistant Professor Department of Nephrology, NRI Medical College and Hospital, Chinakakani, Guntue, Andhra Pradesh, India

Dr. Praveen Kumar Kolla Professor, Department of Nephrology, Narayana Medical College, Nellore, Andhra Pradesh, India

Dr. Raghavendra Sadineni Assistant Professor, Department of Nephrology, Narayana Medical College, Nellore, Andhra Pradesh, India

Corresponding Author: Dr. K Vara Prasada Rao Associate Professor, Department of Nephrology, Narayana Medical College, Nellore, Andhra Pradesh, India

\section{Effect of dialysate sodium concentration on interdialytic weight gain in hemodialysis patients}

\author{
Dr. Spandana Vuyyuru, Dr. K Vara Prasada Rao, Dr. Moturu Venkata \\ Viswanath, Dr. Praveen Kumar Kolla and Dr. Raghavendra Sadineni
}

DOI: $\underline{\text { https://doi.org/10.22271/27069567.2021.v3.i2b.270 }}$

\begin{abstract}
Aim: To study the effect of individualized dialysate sodium on interdialytic weight gain and blood pressure.

Methodology: It was a Prospective study. Duration: 2 years from January 2019 to December 2020. In this study, about 50 patients on maintenance hemodialysis for at least six weeks and IDWG more than $3 \mathrm{~kg}$ were enrolled. Patients receiving maintenance hemodialysis in Narayana Medical College and Hospital Nellore were enrolled in the present study.

Results: Fifty patients who met the inclusion criteria were enrolled in our study. These include $33(66 \%)$ males and $17(34 \%)$ females with a mean age of 51.58 years. The mean duration of HD in our study was 16.92 months. The most common cause of ESRD in our study was Diabetic Nephropathy (54\%); other causes include Chronic Glomerular Nephritis (18\%), Chronic Interstitial Nephritis (8\%), and CKD of unknown cause in $20 \%$ of patients. Dialysate sodium in standard phase (Phase I) was kept constant at $138 \mathrm{meq} / \mathrm{L}$, whereas mean dialysate sodium in individualized sodium Phase (Phase II), calculated by multiplying mean pre-HD sodium by 0.95 (Donnan coefficient), was $128 \mathrm{meq} / \mathrm{L}$. The Mean pre-HD sodium level was almost similar in both phases, but there was a $3 \mathrm{meq} / \mathrm{L}$ difference in the mean post-HD sodium level in standard and individualized phases. There was a significant reduction in mean IDWG by $0.63 \mathrm{~kg}$ in Phase II, and there was also a significant reduction in mean UF requirement by $0.55 \mathrm{~kg}$ in the individualized sodium phase (Phase II) when compared with the standard phase (Phase I). There was a significant reduction in post-HD diastolic blood pressure by $3 \mathrm{mmHg}$ in the individualized sodium phase (Phase II) compared with the standard phase (Phase II). There was a reduction in intradialytic complications in Phase II when compared with Phase I. Despite the low dialysate sodium concentrations, there was no increase in hypotension episodes in the individualized sodium phase (Phase II).
\end{abstract}

Conclusion: We conclude that individualizing dialysate sodium in hemodialysis patients significantly reduced interdialytic weight gain, ultrafiltration requirement, and blood pressure.

Keywords: dialysate sodium, chronic glomerular nephritis, blood pressure, diabetic nephropathy

\section{Introduction}

Interdialytic weight gain (IDWG) is the major problem in hemodialysis patients. It is associated with increased morbidity and mortality. The major extracellular cation in the body is Sodium. It determines the serum osmolarity and extracellular fluid (ECF) content ${ }^{[1]}$. Fluid overload occurs mainly due to excessive Sodium in the body. In hemodialysis patients, sodium entry occurs through dietary intake, saline infusions, and dialysis fluid during hemodialysis.

At present, all patients undergoing maintenance hemodialysis in our institute and most centers worldwide were dialyzed with standard dialysate sodium of $138 \mathrm{meq} / \mathrm{L}$ irrespective of their serum sodium values. Dialysis patients have individualized osmolarity and sodium levels known as osmolar and sodium set point, respectively, and are unique for each individual ${ }^{[2-5]}$. If the dialysate sodium concentration is more than the patient's plasma sodium level, it causes sodium gain during dialysis, and the total body sodium increases.

A lower sodium setpoint may be seen in these patients, and more Sodium is being added to their body with each hemodialysis (HD) session. It leads to increased thirst, excessive fluid intake and finally, interdialytic weight gain and high blood pressure. Prolonged fluid overload leads to uncontrolled blood pressure, left ventricular hypertrophy and increased cardiovascular morbidity and mortality. 
Theoretically, during hemodialysis, it is advantageous to use individualized dialysate sodium and avoid excess sodium in the body.

Many studies have been done worldwide on individualized sodium prescriptions in HD patients, but the results have been inconsistent. In India, only a few studies were done regarding whether individualized sodium prescription reduces morbidity and mortality in maintenance hemodialysis patients (MHD) patients.

We aim to study whether individualized sodium profiling in dialysis patients will have beneficiary effects on IDWG, blood pressure and reduce HD-related adverse effects.

\section{Aims and Objectives \\ Aims}

To study the effect of individualized dialysate sodium on interdialytic weight gain and blood pressure.

\section{Objectives of the study}

To evaluate the beneficial effects of individualized dialysate sodium.

To study the effect of individualized dialysate sodium on intradialytic complications.

\section{Materials and methods \\ Type of study: Prospective study.}

Duration: 2 years from January 2019 to December 2020.

Place: Department of Nephrology, Narayana Medical College, Nellore Sample size: 50 patients.

In this prospective study, about 50 patients on maintenance hemodialysis for at least six weeks and IDWG more than $3 \mathrm{~kg}$ were enrolled. Patients receiving maintenance hemodialysis in Narayana Medical College and Hospital Nellore were enrolled in the present study.

The study was performed in two different phases and each subject was being used as their own control. In the first phase (Standard Dialysate Sodium phase-Phase I), patients were subjected to 12 consecutive HD sessions with a standard dialysate sodium concentration of $138 \mathrm{mEq} / \mathrm{L}$ (standard concentration in our dialysis unit). Pre and postHD plasma sodium concentrations were determined for each patient during each dialysis session. In the second phase of the study (Individualized Dialysate Sodium phase-Phase II), patients were subjected again to 12 consecutive HD sessions with individualized dialysate sodium value (mean of pre-HD sodium concentration multiplied by Donnan coefficient of 0.95). Pre and post-HD sodium values, interdialytic weight gain, Pre and Post HD systolic and diastolic BP, and major HD related complications (Pulmonary edema, intradialytic hypertension and hypotension) during both phases were assessed.

All patients were counseled regarding dietary salt restriction, fluid restriction, adequate protein intake, and compliance with antihypertensive medications.

\section{Inclusion criteria}

- Age > 18yrs

- Patients with IDWG $>3 \mathrm{~kg}$.

- Patients on maintenance hemodialysis for at least six weeks.

\section{Exclusion criteria}

- Patients with an expected life expectancy of less than six months.

- Pre-HD sodium $<130 \mathrm{mEq} / \mathrm{L}$ or $>140 \mathrm{mEq} / \mathrm{L}$ at the time of recruitment.

- Patients with concomitant illnesses that may limit or contraindicate study procedures and follow-up (e.g., frequent intradialytic hypotension requiring resuscitation, hereditary or infiltrative cardiomyopathies, severe aortic valvular disease).

- Failure to give informed consent

Data was entered in MS-Excel and analyzed in SPSS V25. Descriptive statistics were represented with percentages and mean with SD (Standard deviation). Independent t-test, Paired t-test was calculated. P-value $<0.05$ is considered as statistically significant.

\section{Observation and results}

50 patients were included in the study, and the mean age was 51.58 years.

Table 1: Age distribution

\begin{tabular}{|c|c|c|c|c|c|}
\hline Variable & N & Minimum & Maximum & Mean & SD \\
\hline Age & 50 & 20 & 83 & 51.58 & 13.65 \\
\hline
\end{tabular}

The majority of the patients were males $(66 \%)$.

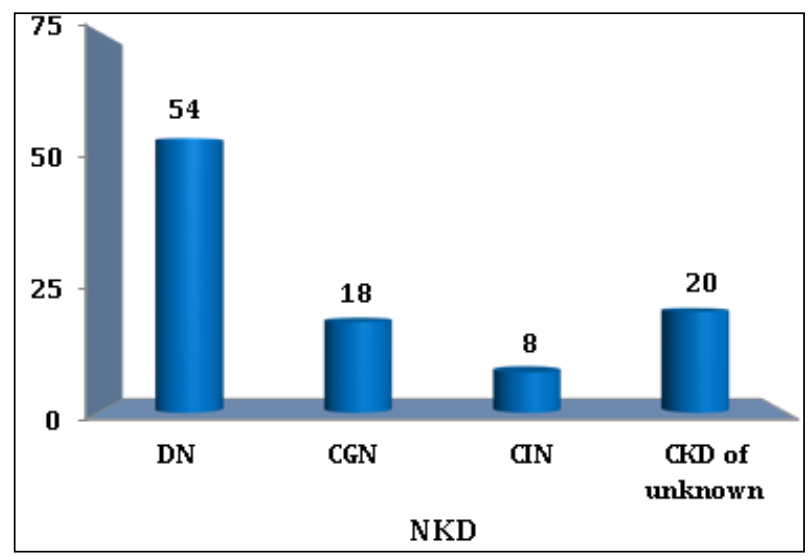

Graph 1: Graph on Frequency of Native Kidney Disease

The most common cause of ESRD in this study was Diabetic nephropathy. The cause of ESRD was diabetic nephropathy (DN) in 27 patients (54\%), chronic glomerulonephritis (CGN) in 9 patients $(18 \%)$, chronic interstitial nephritis (CIN) in 4 patients (8\%), and etiology is not known in 10 patients (20\%).

Table 2: Duration of HD

\begin{tabular}{|c|c|c|c|c|c|}
\hline Variable & N & Minimum & Maximum Mean & SD \\
\hline Duration of HD Upto Phase-1 & 50 & 8 & 50 & 16.92 & 8.60 \\
\hline
\end{tabular}

The mean duration of HD was 16.92 months ranging from 8 to 50 months.

Table 3: Effect on serum sodium

\begin{tabular}{|c|c|c|c|c|c|}
\hline \multirow{2}{*}{ Variable } & \multicolumn{2}{|c|}{ Phase-I } & \multicolumn{2}{c|}{ Phase-II } & \multirow{2}{*}{ P-value } \\
\cline { 2 - 5 } & Mean & SD & Mean & SD & \\
\hline Pre HD Na & 135.24 & 2.25 & 135.60 & 2.11 & $<0.001$ \\
\hline Post HD Na & 137.70 & 2.28 & 134.00 & 1.83 & $<0.001$ \\
\hline P-value & \multicolumn{2}{|c|}{$<0.001$} & \multicolumn{2}{c|}{$<0.001$} & \\
\hline
\end{tabular}




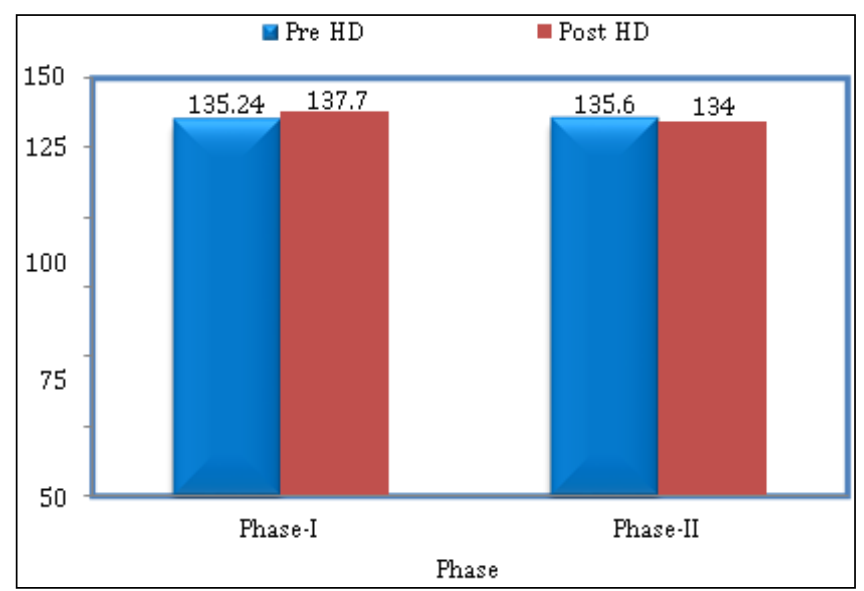

Graph 2: Effect on serum sodium

There was a statistically significant reduction in Post HD serum sodium in the Individualized dialysate sodium phase.

Table 4: Dialysate sodium

\begin{tabular}{|c|c|c|c|c|c|}
\hline \multirow{2}{*}{ Variable } & \multicolumn{2}{|c|}{ Phase-I } & \multicolumn{2}{c|}{ Phase-II } & \multirow{2}{*}{ P-value } \\
\cline { 2 - 5 } & Mean & SD & Mean & SD & \\
\hline Dialysate Na & 138.00 & 0.00 & 128.24 & 2.25 & $<0.001$ \\
\hline
\end{tabular}

Table 5: Effect on IDWG

\begin{tabular}{|c|c|c|c|c|c|}
\hline \multirow{2}{*}{ Variable } & \multicolumn{2}{|c|}{ Phase-I } & \multicolumn{2}{c|}{ Phase-II } & \multirow{2}{*}{ P-value } \\
\cline { 2 - 5 } & Mean & SD & Mean & SD & \\
\hline Interdialytic Wt. gain & 3.97 & 0.52 & 3.34 & 0.44 & $<0.001$ \\
\hline
\end{tabular}

Mean Interdialytic weight gain (IDWG) was $3.97 \mathrm{~kg}$ in Phase I and $3.34 \mathrm{~kg}$ in Phase II.

Table 6: Effect on Ultrafiltration

\begin{tabular}{|c|c|c|c|c|c|}
\hline \multirow{2}{*}{ Variable } & \multicolumn{2}{|c|}{ Phase-I } & \multicolumn{2}{c|}{ Phase-II } & \multirow{2}{*}{ P-value } \\
\cline { 2 - 5 } & Mean & SD & Mean & SD & \\
\hline UF & 3.80 & 0.34 & 3.25 & 0.46 & $<0.001$ \\
\hline
\end{tabular}

Mean Ultra filtration (UF) was $3.8 \mathrm{~kg}$ in Phase I and 3.25 kilograms in Phase II.

Table 7: Effect on Systolic blood pressure

\begin{tabular}{|c|c|c|c|c|c|}
\hline \multirow{2}{*}{ Variable } & \multicolumn{2}{|c|}{ Phase-I } & \multicolumn{2}{c|}{ Phase-II } & \multirow{2}{*}{ P-value } \\
\cline { 2 - 5 } & Mean & SD & Mean & SD & \\
\hline Pre HD SBP & 146.40 & 16.75 & 138.80 & 12.39 & $<0.001$ \\
\hline Post HD SBP & 143.40 & 15.60 & 135.20 & 11.11 & $<0.001$ \\
\hline P-value & \multicolumn{2}{|c|}{0.27} & \multicolumn{2}{c|}{0.06} & \\
\hline
\end{tabular}

Mean pre-systolic BP (SBP) was $146.4 \mathrm{~mm} \mathrm{Hg}$ and 138.8 $\mathrm{mmHg}$ in Phase I and Phase II, respectively. There was a statistically significant reduction in pre HD systolic BP.

There was a statistically significant reduction in post HD systolic BP in phase II.

Table 8: Effect on Diastolic BP (DBP)

\begin{tabular}{|c|c|c|c|c|c|}
\hline & \multicolumn{2}{|c|}{ Phase-I } & \multicolumn{2}{c|}{ Phase-II } & \multirow{2}{*}{ P-value } \\
\cline { 2 - 5 } & Mean & SD & Mean & SD & \\
\hline Pre HD DBP & 83.80 & 7.25 & 82.00 & 7.28 & 0.1 \\
\hline Post HD DBP & 85.00 & 7.07 & 82.00 & 7.00 & 0.01 \\
\hline P-value & \multicolumn{2}{|c|}{0.28} & \multicolumn{2}{c|}{1.00} & \\
\hline
\end{tabular}

Mean post HD diastolic BP in Phase I and Phase II was 85 $\mathrm{mmHg}$ and $82 \mathrm{~mm} \mathrm{Hg}$ and was statistically significant $(\mathrm{P}-$ value 0.01 ). There was no statistically significant reduction in pre HD diastolic BP in phase II compared to phase 1.

Table 9: Frequency of Complications

\begin{tabular}{|c|c|c|c|c|}
\hline \multirow{2}{*}{ Complications } & \multicolumn{2}{|c|}{ Phase-1 } & \multicolumn{2}{c|}{ Phase-2 } \\
\cline { 2 - 5 } & Frequency & Percent & Frequency & Percent \\
\hline No & 23 & 46 & 37 & 74 \\
\hline Intradialytic HTN & 17 & 34 & 8 & 16 \\
\hline Acute Pulmonary Edema & 7 & 14 & 2 & 4 \\
\hline Intradialytic hypotension & 3 & 6 & 3 & 6 \\
\hline Total & 50 & 100 & 50 & 100 \\
\hline
\end{tabular}

In Phase I, intradialytic hypertension (HTN) was present in 17 patients $(34 \%)$, acute pulmonary edema in 7 patients (14\%), and intradialytic hypotension in 3 patients $(6 \%)$.

In Phase II, intradialytic hypertension (HTN) was present in 8 patients (16\%), acute pulmonary edema in 2 patients $(4 \%)$, and intradialytic hypotension in 3 patients $(6 \%)$.

There was a reduction in pulmonary edema and intradialytic hypertensive episodes in phase II, and this was not associated with increased incidence of intradialytic hypotension.

\section{Discussion}

Sodium is the predominant determinant of serum osmolarity, even in uremic patients. It determines intracellular-intravascular fluid distribution, thirst, and blood pressure. Hemodialysis patients have an individualized, highly conserved, and constant pre-dialysis plasma sodium concentration. When extra sodium is added to the body during HD, it increases the thirst and fluid intake to maintain the sodium and osmolar set points. It results in excess weight gain leading to increased extracellular fluid volume and thus higher blood pressure.

Flanigan's study showed that dialysis patients have a relatively constant sodium set point, ranging from 132 to $144 \mathrm{meq} / \mathrm{L}$ in different patients. Their pre-dialysis to postdialysis sodium increased by $2.3-3.6 \mathrm{mEq} / \mathrm{L}$ when dialyzed with $140 \mathrm{meq} / \mathrm{L}$ sodium dialysate ${ }^{[6]}$.

In dialysis, the most common cause of death is due to cardiovascular disease. It occurs primarily due to arrhythmias, and left ventricular hypertrophy (LVH) is the crucial contributing factor associated with this. LVH leads to activation of myocardial fibrotic pathways, leading to stiffened myocardium prone to dilated cardiomyopathy and conduction defects ${ }^{[7]}$. Some studies showed that with improvements in ECF volume and BP control, regression of left ventricular mass occurs. Hence, individualizing the dialysate sodium helps reduce fluid overload in HD subjects and thus reduces the incidence of LVH.

The main findings in our study were a reduction in Interdialytic weight gain, Ultrafiltration, and decreases in pre and post hemodialysis systolic blood pressure in the individualized dialysate sodium phase(Phase II) when compared with the standard phase(Phase I).

Post HD diastolic BP was significantly reduced in the individualized phase. There was no significant difference in pre-HD Diastolic Blood Pressure between the standard phase (Phase I) and the individualized sodium phase (Phase II). There was a significant reduction in the number of dialysis-related complications between standard and individualized phases. Despite the low sodium concentrations used during an individual phase, there was no increase in hypotensive episodes. All patients were on 
antihypertensive drugs and the dose of antihypertensive drugs were reduced in 3 patients. In our study, a mean preHD sodium level of $135.24 \mathrm{meq} / \mathrm{L}$ was seen in the standard Phase (Phase I). The Mean of pre-HD sodium was multiplied with a correction factor of 0.95 (Donnan Coefficient) to get the dialysate sodium value. This will result in eunatremic dialysis. Gibbs-Donnan effect in hemodialysis occurs due to negatively charged nondiffusable plasma proteins to create an electric field that attracts sodium and reduces the diffusion of sodium from plasma across the dialysis membrane.

In our study, there was no much difference in pre-HD sodium between Standard (Phase I) and Individualized dialysate sodium (Phase II) (135.24 $\mathrm{mEq} / \mathrm{L}$ and 135.60 $\mathrm{mEq} / \mathrm{L})$, respectively. This concurs with the concept of a fixed sodium set point in dialysis patients. But there was a $3 \mathrm{meq} / \mathrm{L}$ difference in the post-HD sodium level in standard and individualized phases $(137.70 \mathrm{meq} / \mathrm{L}$ and $134.0 \mathrm{meq} / \mathrm{L})$, respectively.

Several studies showed that dialysate sodium prescriptions individualized to each patient's sodium set point will be beneficial but in differing aspects. De Paula et al. studied 27 HD patients. They reported that reduction of dialysate sodium to as low as pre-dialysis plasma sodium concentration (individualized isometric sodium) significantly found a decrease in interdialytic thirst scores, interdialytic weight gain, pre HD blood pressure, and episodes of intradialytic hypotension in the individualized sodium phase compared with the standard phase ${ }^{[8]}$. Similar results were seen in our study also. There was a significant decrease in IDWG in the individualized phase, and there was no increased incidence of intradialytic hypotension. However, thirst scores were not included in this study.

Aramreddy et al. reported a case series of thirteen patients undergoing thrice-weekly in-center hemodialysis with an individualized dialysate sodium prescription. The dialysate sodium concentration was $2 \mathrm{meq} / \mathrm{L}$ lower than average plasma sodium over the preceding three months. They found that individualized reduction of dialysate sodium reduces IDWG without significantly increased frequency of cramps or hypotension ${ }^{[9]}$. Our study found similar results with individualized sodium with a significant decrease in IDWG and no increased frequency of hypotensive episodes.

Similar results were obtained by Elshahawy et al., ${ }^{[10]}$ who studied 40 stable chronic HD patients in a single-blinded crossover design. Individualized dialysate sodium concentration was associated with decreased IDWG, intradialytic hypotension, and related symptoms with better BP control in stable chronic HD patients ${ }^{[10]}$. Our study also found a significant reduction in Pre and post HD systolic BP with no increase in major adverse events.

In a recent study by Sayarlioglu et al. ${ }^{[11]}$ in 18 hemodialysis patients, they used the pre-dialysis sodium as a reference to set the dialysate sodium concentration. For patients with pre-HD sodium less than $137 \mathrm{mEq} / \mathrm{L}$, the dialysate sodium was modified to $135 \mathrm{mEq} / \mathrm{L}$, and for patients with pre-HD sodium over $137 \mathrm{mEq} / \mathrm{L}$, the dialysate sodium was modified to $137 \mathrm{mEq} / \mathrm{L}$. After eight weeks, they found that reducing dialysate sodium resulted in a significant decrease in IDWG $(1.8 \pm 0.6 \mathrm{~kg}$ versus $2.5 \pm 1 \mathrm{~kg})$, pre-HD systolic blood pressure (SBP) $(151.7 \pm 17.7 \mathrm{mmHg}$ versus $179.7 \pm 24.8$ $\mathrm{mmHg})$, pre-HD diastolic blood pressure (DBP) $(93.1 \pm 10.5$ $\mathrm{mmHg}$ versus $100.6 \pm 12.8 \mathrm{mmHg}$ ), post-HD SBP (132.3 \pm $16.4 \mathrm{mmHg}$ versus $141.4 \pm 28.8 \mathrm{mmHg}$ ). In our study, by using individualized dialysate sodium, there was a reduction in IDWG, pre HD systolic BP, post HD systolic BP and post HD diastolic BP. There was also a reduction in pre-HD diastolic BP in the individualized dialysate sodium phase, but it is not statistically significant.

Sanjeev et al. studied 50 patients on maintenance hemodialysis for at least three months with intradialytic hypertensive episodes. The study was conducted in 2 phases. In the first phase, patients were dialyzed for eight consecutive sessions with standard dialysate sodium of 140 $\mathrm{mEq} / \mathrm{L}$ and in the second phase with dialysate sodium of $136 \mathrm{mEq} / \mathrm{L}$. They concluded that intradialytic hypertensive episodes and post HD blood pressure were reduced in the reduced dialysate sodium phase. In our study also the intradialytic hypertensive episodes were reduced in the individualized dialysate sodium phase.

Raimann JG et al. concluded that the sodium gradient in maintenance hemodialysis patients was significantly and positively correlated with IDWG. The changes in dialysate sodium concentration in dialysis may affect the IDWG of MHD patients. Too low or too high sodium gradient may affect IDWG, resulting in fluid overload and further complications. Therefore, the individual dialysate sodium prescription may improve the fluid load in MHD patients [12].

In a recent study, the dialysate sodium level was individualized in around 27 stable patients. They reduced the dialysate sodium concentration on average by three $\mathrm{mEq} / \mathrm{L}$ to achieve a sodium gradient of $0 \mathrm{mEq} / \mathrm{L}$. At about three weeks of follow-up, thirst scores had improved, interdialytic weight gain was reduced, and blood pressure was also improved in the subgroup of patients with uncontrolled hypertension.

Similar to our study, a study by Kawain et al. found that individualized dialysate sodium had a beneficial effect on IDWG. There was a significant reduction in IDWG and blood pressure without an increase in the frequency of adverse effects ${ }^{[13]}$.

In the DISO trial, they observed a significant reduction in IDWG during the individualized sodium phase. In the individualized phase, the thirst score was also significantly lower. Pre-and post-hemodialysis systolic blood pressures were significantly reduced by a mean value of $4 \mathrm{mmHg}$ during the individualized sodium phase compared with the standard dialysate sodium phase. But there was no difference in diastolic BP. The number of intradialytic hypertension episodes was significantly reduced when patients received individualized sodium dialysis. Despite the low sodium concentrations used during hemodialysis, there was no increase in hypotension, symptoms such as muscle cramps, or requirement of interventions during the individualized phase. The necessity of antihypertensive drugs during individualized sodium dialysis was decreased in $19 \%$ of patients ${ }^{[14]}$. Our study also showed a significant reduction in IDWG, Pre and post HD systolic BP, and post HD diastolic BP in the individualized dialysate sodium phase compared to the standard dialysate sodium phase. Post HD sodium was reduced by a mean of $3 \mathrm{mEq} / \mathrm{L}$, and pre $\mathrm{HD}$ systolic $\mathrm{BP}$ was reduced by a mean of $7 \mathrm{~mm} \mathrm{Hg}$ in the individualized sodium phase.

\section{Conclusion}

- We conclude that individualizing dialysate sodium in hemodialysis patients significantly reduced interdialytic 
weight gain, ultrafiltration requirement, and blood pressure.

- There was a decrease in pulmonary edema and intradialytic hypertension episodes with individualized dialysate sodium.

- Even though low dialysate sodium was used in the individualized phase, there was no increase in intradialytic hypotension events.

- Hence individualized dialysate sodium is beneficial to hemodialysis patients.

\section{References}

1. Flanigan MJ. Role of sodium in hemodialysis. Kidney Int 2000;58:S72-8.

2. De Paula FM, Peixoto AJ, Pinto LV, Dorigo D, Patricio PJ, Santos SF. Clinical consequences of an individualized dialysate sodium prescription in hemodialysis patients. Kidney Int 2004;66:1232-1238.

3. Gotch FA, Lam MA, Prowitt M, Keen M. Preliminary clinical results with sodium volume modeling of hemodialysis therapy. Proc Clin Dial Transplant Forum 1980;10:12-17.

4. Sayarlioglu H, Erkoc R, Tuncer M, Soyoral Y, Esen R, Gumrukcuoglu HA. Effects of low sodium dialysate in chronic hemodialysis patients: an echocardiographic study. Ren Fail 2007;29:143-146.

5. Suckling RJ, Swift PA, He FJ, Markandu ND, MacGregor GA. Altering plasma sodium concentration rapidly changes blood pressure during hemodialysis. Nephrol Dial Transplant 2013;28:2181-2186.

6. Flanigan MJ. How should dialysis fluid be individualized for the chronic hemodialysis patient? Sodium. Semin Dial 2008;21:226-9.

7. Ritz E. Left ventricular hypertrophy in renal disease: Beyond preload and afterload. Kidney Int 2009;75:771-3.

8. De Paula FM, Peixoto AJ, Pinto LV, Dorigo D, Patricio PJ, Santos SF. Clinical consequences of an individualized dialysate sodium prescription in hemodialysis patients. Kidney Int 2004;66:1232-8.

9. Arramreddy R, Sun SJ, Munoz Mendoza J, Chertow GM, Schiller B. Individualized reduction in dialysate sodium in conventional in-center hemodialysis: Dialysate sodium in conventional HD. Hemodial Int 2012;16:473-80.

10. Elshahawy Y, Sany D, Shawky S. Outcome of individualized dialysate sodium concentration for hemodialysis patients. Saudi J Kidney Dis Transplant 2013;24:507-13.

11. Sayarlioglu H, Erkoc R, Tuncer M, Soyoral Y, Esen R, Gumrukcuoglu HA et al. Effects of low sodium dialysate in chronic hemodialysis patients: an echocardiographic study. Ren Fail 2007;29:143-146.

12. Raimann JG, Ficociello LH, Usvyat LA et al. Effects of dialysate sodium $(\mathrm{Na}+)$ alignment in chronic hemodialysis (HD) patients: retrospective cohort study from a quality improvement project. BMC Nephrology 2018;19:75.

13. Kawin Gunasekaran, Ramprasad Elumalai E, Jayakumar $\mathrm{M}$ et al. Effects of Individualized Dialysate Sodium in Hemodialysis, J Res Med Dent Sci 2021;9(1):50-56.

14. Radhakrishnan RC, Varughese S, Chandra A et al. Effects of individualized dialysate sodium prescription in hemodialysis-Results from a prospective interventional trial. Indian J Nephrol 2020;30:3-7. 